\title{
Cuidado interdisciplinar em uma clínica ensino: Percepções e processos de trabalho
}

\author{
Interdisciplinary care in a teaching clinic: Perceptions and work processes \\ Atención interdisciplinar em uma clínica docente: Percepciones y processos de trabajo
}

Recebido: 23/03/2021 | Revisado: 29/03/2021 | Aceito: 01/04/2021 | Publicado: 12/04/2021

Tauane Letícia Pinto Zanelli

ORCID: https://orcid.org/0000-0001-9955-4836 Centro Universitário da Fundação Hermínio Ometto, Brasil

E-mail: tauanezanelli@gmail.com

Camila Binotti

ORCID: https://orcid.org/0000-0003-3376-9433

Hospital e Maternidade Madre Theodora, Brasil

E-mail: camilabinotti.cb@gmail.com

Natanaellin Eydiane da Silva Begnami

ORCID: https://orcid.org/0000-0002-3959-0082

Centro Universitário da Fundação Hermínio Ometto, Brasil

E-mail: enf.natanaellin@gmail.com

Maria Giovana Borges Saidel

ORCID: https://orcid.org/0000-0002-3259-1760

Universidade Estadual de Campinas, Brasil E-mail:mgsaidel@unicamp.br

Luiz César Gerotto Júnior

ORCID: https://orcid.org/0000-0001-8832-5560

Centro Universitário da Fundação Hermínio Ometto, Brasil

E-mail: luizcesargerotto@hotmail.com

Clarice Santana Milagres

ORCID: https://orcid.org/0000-0002-9164-4340

Centro Universitário da Fundação Hermínio Ometto, Brasil

E-mail: claricemilagres01@gmail.com

\section{Resumo}

Objetivo: compreender as percepções de estudantes de graduação que atuam em uma clínica ensino sobre o cuidado interdisciplinar e os processos de trabalho das equipes. Método: qualitativo em uma clínica de ensino de uma instituição de ensino superior. A amostra foi constituída por alunos de graduação em enfermagem, psicologia e fisioterapia, a partir de entrevista semiestruturada, observação participante e diário de campo. Utilizou-se a análise de conteúdo de Bardin e o referencial teórico, a Teoria da Conservação de Energia e da Enfermagem holística de Myra Levine Resultados: foram obtidas duas categorias temáticas: percepções sobre os processos de trabalho desenvolvidos dentro da clínica pelas equipes e; percepção de um cuidado ideal. Os cursos envolvidos ainda não superaram os modelos tradicionais constituídos em núcleos, no qual a aprendizagem compartilhada ainda é segregada. Conclusão: adequação ao perfil profissional da área da saúde em prol de um trabalho em equipe e na prática interprofissional.

Palavras-chave: Equipe de assistência ao paciente; Saúde holística; Assistência integral à saúde; Integralidade em saúde; Práticas interdisciplinares.

\begin{abstract}
Objective: to understand the perceptions of undergraduate students who work in a teaching clinic on interdisciplinary care and team work processes. Method: qualitative in a teaching clinic of a higher education institution. The sample consisted of undergraduate students in nursing, psychology and physiotherapy, based on semi-structured interviews, participant observation and field diaries. Bardin's content analysis and the theoretical framework, Myra Levine's Theory of Energy Conservation and Holistic Nursing were used. Results: two thematic categories were obtained: perceptions about the work processes developed within the clinic by the teams and; perception of ideal care. The courses involved have not yet surpassed the traditional models made up of nuclei, in which shared learning is still segregated. Conclusion: adequacy to the professional profile of the health area in favor of teamwork and in interprofessional practice.
\end{abstract}

Keywords: Patient care team; Holistic health; Comprehensive health care; Integrality in health; Interdisciplinary placement.

\section{Resumen}

Objetivo: comprender las percepciones de los estudiantes de pregrado que laboran en una clínica docente sobre la atención interdisciplinaria y los procesos de trabajo en equipo. Método: cualitativo en una clínica de enseñanza de una institución de educación superior. La muestra estuvo conformada por estudiantes de pregrado en enfermería, psicología y fisioterapia, con base 
en entrevistas semiestructuradas, observación participante y diarios de campo. Se utilizó el análisis de contenido de Bardin y el marco teórico, Teoría de Conservación de Energía y Enfermería Holística de Myra Levine Resultados: se obtuvieron dos categorías temáticas: percepciones sobre los procesos de trabajo desarrollados dentro de la clínica por los equipos y; percepción del cuidado ideal. Los cursos involucrados aún no han superado los modelos tradicionales compuestos por núcleos, en los que el aprendizaje compartido sigue segregado. Conclusión: adecuación al perfil profesional del área de salud a favor del trabajo en equipo y en la práctica interprofesional.

Palabras clave: Grupo de atención al paciente; Salud holística; Atención integral de salud; Integralidad en salud; Prácticas interdisciplinarias.

\section{Introdução}

O processo de trabalho em equipe pode ser definido como pluridisciplinariedade, multidisciplinaridade, transdisciplinaridade e interdisciplinaridade, e, apresenta como objetivo a obtenção de impactos sobre os diferentes fatores que interferem no processo saúde-doença. Para tanto há diversas interlocuções na saúde, e, por mais complexas e profundas que sejam, não são capazes suprir a extensa problemática humana, que apresentam métodos científicos de abordagens das relações humanas como ponto fundamental para a busca pela integralidade do indivíduo ao associá-lo ao meio social, político, cultural, biológico, comportamental, dentre outras (Peduzzi et al., 2020).

Na organização do trabalho em equipe, a pluridisciplinaridade é caracterizada como o sistema de um único nível e objetivos múltiplos, com cooperação e ausência de coordenação. A multidisciplinaridade trata da execução de várias disciplinas sem relações entre si, enquanto a transdisciplinaridade é definida como um trabalho coletivo que compartilha as estruturas conceituais a fim de construir teorias, conceitos e abordagens juntos para tratar problemáticas comuns. Por fim, a interdisciplinaridade é uma etapa contínua, constituída por profissionais que trabalham juntos com pontos de vista paralelos, avançando para combinar as ideias, e, desta forma, alcançar um processo interdisciplinar. Para quando há essa aproximação de um ponto de fusão e integração dos saberes de cada um, consegue-se chegar a uma perspectiva holística (Japiassu, 1976; Wilk et al., 2020; Costa, 2017).

As diferentes definições do processo de trabalho em equipe podem ser modificadas conforme o trabalho proposto, ou seja, variar conforme o objetivo da unidade de trabalho e assistência (Agreli, 2017). Profissionais de saúde que configuram clínicas de ensino apresentam a interdisciplinaridade, enquanto a assistência terciária, muitas vezes, apresenta as demais definições deste processo de trabalho (Wilk et al., 2020). Instituições que já definem os papéis de cada ente envolvido e a organização do processo de trabalho demonstram o impacto da EIP na formação dos profissionais. (Lewgoy, Kuplich, Machado, Faber \& Fagundes, 2019).

As teorias de enfermagem contribuem na ampliação conceitual de boas práticas de enfermagem, assim como contribuem ao fundamentar o processo de trabalho em equipe e suas definições (Salviano et al., 2016; Oliveira et al., 2019). O referencial teórico que destaca o cuidado integral oferecido para o indivíduo pode ser analisado segundo a Teoria da Conservação de Energia e da Enfermagem Holística de Myra Levine, que propõe uma enfermagem clínica, entendendo o paciente como corpo-mente, ou seja, um "todo" com interação ao meio dinâmico. É nesta visão humanística que o conceito da interdisciplinaridade é alcançado. Vale ressaltar que essa teoria, ao ser aplicada junto aos métodos de trabalho, respeitando princípios, crenças e valores do indivíduo, também é capaz de apresentar particularidades e integralidade do indivíduo, colocando-o como protagonista de seu meio, ao atuar de forma consciente e responsável em defesa do trabalho realizado (Levine, 1996).

Diante do exposto, este estudo tem como pergunta norteadora: qual a percepção de estudantes de graduação sobre os processos de trabalho interdisciplinar em uma clínica ensino? E o objetivo foi compreender as percepções de estudantes de graduação que atuam em uma clínica ensino sobre o cuidado interdisciplinar e os processos de trabalho das equipes. 


\section{Metodologia}

\subsection{Delineamento da Pesquisa}

Estudo de abordagem qualitativa que utilizou como método a pesquisa convergente assistencial que tem como característica principal manter, durante todo o seu processo, uma estreita relação com a situação da prática assistencial, com a intenção de encontrar os principais focos a serem trabalhados, como a soluções para problemas, realização mudanças, ou mesmo introduzir inovações na prática realizada (Trentini, Paim \& Silva, 2017).

Buscou-se adequar as percepções de estudantes de graduação que atuam em uma clínica ensino sobre o cuidado interdisciplinar e os processos de trabalho ao analisar uma abordagem centrada no ser humano, em suas experiências e como atribuem significados únicos a elas.

Nesse tipo de estudo, o foco é direcionado na maximização da percepção do fenômeno, ou seja, da qualidade dos dados coletados e não a quantidade, considerando na análise, as subjetividades dos sujeitos (Oliveira, Baixinho \& Presado, 2019).

\subsection{Local do estudo e População Alvo}

O campo do estudo foi uma clínica de ensino de uma Instituição de Ensino Superior (IES) particular do interior de São Paulo, na qual são realizados atendimentos gratuitos de avaliação e curativo de lesões teciduais, aferição de pressão arterial e glicemia capilar, exame físico, consulta ginecológica, consulta e atendimento em saúde do trabalhador, assistência em ortopedia/postura, neurologia, pneumologia, pediatria, hidroterapia e outras atividades como ergonomia e saúde corporal, avaliação psicológica, psicodiagnóstico e psicoterapia, além de da assistência ao tratamento que acontece junto à equipe interdisciplinar a qual é nomeada de Núcleo Interdisciplinar de Reabilitação (NIR) sendo configurada por professores e alunos de das disciplinas em saúde dos cursos de enfermagem, fisioterapia e psicologia, dos quais promovem aproximadamente 40 atendimentos mensais

A população do estudo foram alunos de graduação enfermagem, psicologia e fisioterapia, que atuam nesta clínica.

Os critérios de inclusão foram: alunos regularmente matriculados na Instituição de Ensino Superior (IES) nos cursos de enfermagem, fisioterapia e psicologia, que desenvolviam a assistência de forma interdisciplinar na Clínica Ensino no período do estudo. Enquanto os critérios de exclusão são os alunos dos cursos de fisioterapia, enfermagem e psicologia que não atuam na assistência interprofissional na clínica ensino.

\subsection{Plano amostral}

O plano amostral foi definido por intencionalidade e fechado por saturação teórica. A amostra por intencionalidade envolve o uso de pessoas mais convenientemente disponíveis como participantes. $\mathrm{O}$ fechamento amostral foi utilizado a saturação teórica. Nesse conceito as entrevistas foram encerradas a partir do momento em que as informações alcançam a redundância (Minayo, 2014).

\subsection{Coleta de dados}

Foi realizado um período de aculturação, no qual as pesquisadoras foram monitoras na clínica durante dez meses. Nesse período foi possível conhecer os processos de trabalho do local. A coleta de dados foi realizada por meio de observação participante, diário de campo e entrevistas em profundidade semiestruturadas com prévio agendamento com a população do estudo. 
A coleta de dados ocorreu de março a junho de 2016, através da observação participante, registro em diário de campo e uma sessão de entrevista com média de 40 minutos de duração, a partir da assinatura do Termo de Consentimento Livre e Esclarecido (TCLE).

A entrevista semiestruturada, método de coleta de dados fundamental nesse estudo, privilegia a obtenção de informações através da fala individual, a qual revela condições estruturais, sistemas de valores, normas e símbolos e transmite, através de um porta-voz, representações de determinados grupos (Minayo, 2014).

\subsection{Análise dos dados}

A análise dos dados foi realizada por meio da Análise de Conteúdo (Minayo, 2014) e utilizando como referencial teórico, a Teoria da Conservação de Energia e da Enfermagem holística de Myra Levin (Levine, 1996) para fundamentar a discussão. A Análise de Conteúdo procura compreender os objetivos dos estudos desenvolvidos. É um meio de expressão do sujeito, no qual o analista busca categorizar as unidades de texto (palavras ou frases) que se repetem, inferindo uma expressão que as representem. Este tipo de análise apresenta uma técnica capaz de proporcionar uma avaliação organizada do texto, ao identificar os temas mais recorrentes e as palavras mais relevantes para ser investigado. Posteriormente, comparar as informações coletadas para se obter uma conclusão que se dá por um tratamento da informação contida nas mensagens dos participantes. A metodologia proposta foi a categorização, inserida na análise de conteúdo, no qual emprega a representatividade, ou seja, uma amostra de represent ação de um universo inicial. Para as três etapas da categorização da análise de conteúdo foram observadas: pré-análise, que consistiu na escolha dos documentos a serem analisados, sistematização de hipóteses e ideias iniciais e criação de categorias de análise; exploração, na qual ocorreu a classificação e categorização do material; e, por fim, tratamento e interpretação dos resultados obtidos (Minayo, 2014).

\subsection{Aspectos éticos}

As entrevistas foram realizadas mediante entrega da carta de apresentação da pesquisa aos usuários, bem como leitura e assinatura do Termo de Consentimento Livre e Esclarecido (TCLE) seguindo a Resolução No 466, de 12 de dezembro de 2012 (Brasil, 2012).

O estudo foi aprovado pelo Comitê de Ética em Pesquisa com Seres Humanos do Centro Universitário da Fundação Hermínio Ometto (FHO) e Plataforma Brasil, sob CAAE: 51255215.9.0000.5385 e aprovação n 1.355 .281 em 08 de dezembro de 2015.

Para manter a privacidade e confidencialidade dos participantes, na apresentação das falas, optou-se por utilizar a letra "E" para alunos da enfermagem, "P" para alunos da psicologia e "F" para os alunos de fisioterapia. Todos foram seguidos do número correspondente à entrevista - por exemplo, à primeira entrevista foi designado o código P1.

\section{Resultados}

Participaram deste estudo um total de dez alunos que foram identificados no estudo com códigos alfanuméricos para manter a privacidade da identidade dos participantes, esses indivíduos compões as equipes que atuam no Núcleo Interdisciplinar de Reabilitação (NIR) da clínica ensino entre os cursos de graduação em enfermagem, psicologia e fisioterapia, dos quais quatro eram do curso de enfermagem, quatro da psicologia e dois da fisioterapia.

A amostra foi composta por nove mulheres e um homem. Em relação à progressão na graduação, dois alunos cursavam o $7^{\circ}$ período e os demais cursavam o $9^{\circ}$ período.

Da análise das transcrições das entrevistas obtidas emergiram duas categorias temáticas: 1) percepções sobre os processos de trabalho desenvolvidos dentro da clínica pelas equipes e; 2) percepção de um cuidado ideal. 


\section{1) Percepções sobre os processos de trabalho desenvolvidos dentro da clínica pelas equipes}

Os resultados obtidos das entrevistas mostram que os alunos de graduação dos cursos da saúde elencados buscam conhecer processos de trabalho desenvolvido e apresentam percepções positivas sobre tais processos. Os graduandos em psicologia e fisioterapia, trouxeram em seus depoimentos satisfação à assistência prestada:

Aqui na clínica eu vejo que todo trabalho tem bastante empenho de todo mundo das equipes. (P-1)

É muito bacana como está hoje o trabalho aqui na clínica porque já me falaram que não era dessa forma, era um tratamento completamente diferente, muito separado, sem entrosamento. (F-2)

Em diferentes trechos os graduandos relatam não sentirem dificuldades em realizar suas atividades, mesmo com intervenções das outras diferentes equipes. Quando abordam a existência de dificuldades, as mesmas são relatadas como parte de um processo natural do trabalho em equipe, como relatado:

Eu acho que tudo é bem interligado com as três equipes aqui dentro da clínica. Eu não tenho nenhuma dificuldade e funciona bem, mesmo também em cursos diferentes. (F-1)

Então, isso de existir uma dificuldade [...] é uma dificuldade normal, talvez não tenha como ela não existir [...], mas que tem como a gente ir contornando também. Faz parte do trabalho. (P-4)

Os alunos de enfermagem, em sua totalidade relataram que os processos de trabalho na clínica ensino poderiam ser melhor desenvolvidos, uma vez que perceberam e relataram dificuldades em exercer suas atividades com as demais equipes envolvidas na assistência ao usuário:

Bom, na minha percepção, cada equipe trabalha de maneira correta, porém eu acredito que falta trabalhar um pouco mais juntos. Acredito que exista uma postura de resistência por parte das outras equipes. A psicologia no tempo que estive aqui não senti resistência, mas também não senti apoio [...]. Em relação a fisioterapia eu sinto que existe uma resistência por parte deles, são um número muito maior de alunos do que nós, eles ficam um tempo muito maior na clínica do que a gente [...]. (E-3)

Eu acho que necessita de melhorias [...] o curso de fisioterapia predomina sobre o curso de enfermagem, sobre da psico e isso dificulta a interação da equipe que parece só focar em fisioterapia, reabilitação, sei lá. (E-2)

Acerca do modelo de equipe que atuavam na clínica ensino, pode-se observar que um reduzido número de acadêmicos não lembrava e/ou desconheciam a definição e a diferença entre interdisciplinar e multidisciplinar, necessitando explicações didáticas sobre modelos de processos de trabalho em equipe propostos na clínica ensino, assim como esclarecimentos sobre interdisciplinaridade e multidisciplinaridade.

Eu acredito que seja interdisciplinar no sentido que se discute e conversa e faz essa uma atuação única. Então tem uma união de pensamento, de atendimentos, até de intervenções, embora seja muito específico de cada área. Conversando conseguimos pensar numa intervenção que seja ampla no sentido de contemplar as demandas que o sujeito tem. (P-3) 
Eu acredito que aqui na clínica seja um trabalho de equipe interdisciplinar por existir uma conversa entre os profissionais. Não é somente um serviço oferecido onde cada área trabalha separadamente. (P-4)

Aqui o trabalho é multidisciplinar, porque cada um faz a sua parte. Está começando a querer transformar isso em inter, porém eu vejo muito divido ainda, cada um tem a sua função aqui dentro. (E-2)

A proposta da clínica seria interdisciplinar, porém é multidisciplinar, justamente por causa desse cuidado fragmentado. Eu não vejo as equipes trabalhando juntas, e sim, elas trabalham no mesmo lugar, porém cada um faz a sua parte. (E-3)

Eu acho que é mais multi do que inter, porque tem várias equipes atuando no mesmo espaço. Só que eu acho que ainda falta comunicação, mais interação interdisciplinar, mais troca. $(\mathrm{P}-1)$

\section{2) A percepção de um cuidado ideal}

A percepção de um cuidado ideal e integral também pode ser abordado e percebido pelo aluno da psicologia:

Apareceu uma fratura [...] assim não adianta reabilitar só aquela fratura, têm todo um contingente em volta da pessoa que também precisa ser reabilitado né? Não é só aquela fratura, mas como ela vai lidar com aquilo, como que ela vai na casa dela ela lidar com aquilo, aqui como ela lida com aquilo, no social como ela vai lidar com aquilo. (P1)

Mediante ao questionamento acerca dos tratamentos oferecidos pelos acadêmicos, foi analisado a percepção sobre um cuidado integral prestado. Este, por sua vez, divergiu nas falas dos entrevistados quando questionados acerca do mesmo ser integral ou fragmentado:

Acho que ele tem que ser visto como um todo, integral. Porque não adianta eu tratar só uma parte dele. [...]. E aqui é feito desta maneira, integral, mas ainda sim, acabamos focando naquilo que mais aprendemos no curso. (F-1)

Ainda eu acho que está fragmentado. Eu acho que o cuidado está sendo em partes ainda, não está sendo o cuidado como um todo. Cada um parece que enxerga o paciente de acordo com o seu curso. (E-1)

Eu acho que tem casos um pouco mais complexos que às vezes acaba tendo uma atenção um pouco mais fragmentada, pontualmente, em algumas questões. [...]. (P-3)

\section{Discussão}

Os resultados reforçam que a educação interprofissional em saúde pode ser entendida e aplicada em momentos nos quais duas ou mais profissões de saúde entendam de assuntos diversos, aprimorando a qualidade da assistência, assim como a colaboração entre as diferentes profissões. Esta colaboração é fundamental para a construção de uma assistência de qualidade no qual atitudes, conhecimentos, habilidade e comportamentos positivos à uma prática colaborativa pode influenciar a melhor conduta na prática clínica (Almeida \& Silva, 2019; Peduzzi, 2016).

Comparando as falas dos discentes do curso de enfermagem e as dificuldades trazidas por autores, compreende-se a necessidade de ampliação do trabalho em equipe para uma prática interprofissional pautada em comunicação efetiva, junção de ações e sinergia entre os profissionais das diversas áreas. ${ }^{13}$ Entretanto, vale ressaltar que a forma alcançada pela prática interprofissional colaborativa centrada no paciente/usuário também apresenta dificuldades na operacionalização, assim como percebido pelos alunos e corroborada pela literatura (Farias et al., 2018). 
Algumas das dificuldades encontradas na operacionalização do trabalho em equipe interprofissional podem ser destacadas, como a quebra da comunicação, acompanhadas por informações incompletas; rumores, utilização de linguagens especializadas que não são de conhecimento comum, desconhecimento do papel desempenhado pelos demais membros da equipe, desconsiderando, inclusive as experiências e saberes envolvidos nas vivências individuais destes membros e coletivas das equipes; conflitos relacionados à assistência dos cuidados prestados; e indefinições de resultados planejados em projetos assistenciais comuns e alcançados pela equipe (Farias et al., 2018).

O modelo de assistência prestada na clínica ensino é recente, os processos de trabalho envolvendo três graduações da área da saúde é construído continuamente, demandando, portanto, tempo e ajustes necessários a fim de alcançar uma assistência de qualidade aos usuários, assim como aos profissionais e demais envolvidos nas atividades acadêmicas deste campo de atuação.

A percepção de atuação destes acadêmicos, portanto, demonstra anseios por uma colaboração entre os envolvidos que seja não só qualificada, mas humana ao paciente, uma vez que apresentam perspectivas e objetivos pertinentes à uma consolidação do aprendizado aliados às particularidades dos atendimentos. Uma assistência de qualidade prestadas aos usuários deve sobrepor às expectativas de ausência de conflitos e às divergências na equipe, assim como as mais variadas formas de disputas pelo poder que contribuem para as interferências nos processos comunicacionais e de articulação das atividades dos diferentes profissionais envolvidos (Peduzzi, 2016).

A interdisciplinaridade é vista como etapa contínua constituída por profissionais trabalhando juntos para a superação da fragmentação, quando há essa aproximação consegue-se chegar a uma perspectiva holística (Levine, 1996; Farias et al., 2018). A multidisciplinaridade, por sua vez, indica uma execução de várias disciplinas que não apresentam relações entre si, mas estão em um mesmo local e trabalhando isoladamente (Japiassu, 1976; Wilk et al., 2020; Costa, 2017).

Foram construídas várias tentativas para compreensão da relação objeto-conhecimento (Ferraro, Ferri \& Simões, 2017). Quando se tem percepções sobre o objeto a partir de diferentes campos/disciplinas descreve a multidisciplinariedade enquanto a interdisciplinaridade é descrita como a elaboração de estratégias e reflexões para outras compreensões, análise e descrições acerca do objeto em conjunto. Não é tão simples quanto parece ser, há a necessidade de refletir qual estratégia para o processo de trabalho irá atender os objetivos propostos, o contexto que acontece bem como o ambiente, o perfil dos participantes, a abordagem (Ely \& Toassi, 2017). No presente estudo, acredita-se que a dificuldade do entendimento acerca das classificações pelos discentes está em sua incorporação precoce no campo da educação interprofissional, quando a incorporação acontece em um momento mais avançado da graduação é mais proveitoso, visto que poderão demonstrar maior segurança no seu papel profissional (uniprofissional) para, assim atuarem como membros da equipe (interprofissional). Contudo, não há um consenso acerca do melhor momento para ser introduzida a educação interprofissional na graduação, entendendo, portanto, que a inserção nos primeiros anos da formação favorece a redução de efeitos negativos da socialização profissional, por exemplo, os estereótipos hostis, promovendo atitudes e comportamentos positivos a partir da relação com os demais profissionais (Almeida \& Silva, 2019).

Um dos princípios doutrinários que regem o Sistema Único de Saúde (SUS) é a integralidade. A ocorrência do cuidado integral eficaz é consequência da atuação conjunta e colaborativa dos profissionais envolvidos, no qual estratégias de promoção entre os profissionais de forma inter, permita a criação uma visão completa e holística sobre o cuidado, assim como sobre a diversidade de disciplinas das mais diversas profissões relacionadas à atenção à saúde (Almeida \& Silva, 2019; Peduzzi, 2016; Farias et al., 2018).

Em uma abordagem holística, a enfermagem possui modelos teóricos, que apresentam características peculiares, podendo ser trabalhadas independente do modelo teórico. A Teoria da Conservação de Energia e da Enfermagem Holística de Myra Levine, trata de uma proposta de construção de uma enfermagem clínica, no qual entende-se o paciente como corpo físico e mente (Levine, 1996). Os suportes e aconselhamentos para as necessidades dos usuários da clínica ensino, subsidiadas pela 
teoria Holística trazem os subsídios para o cuidado físico, assim como auxiliam na manutenção da integridade daqueles que são assistidos (Peduzzi, 2017). Contudo, ao analisar as falas encontradas neste estudo, anteriores ao referencial teórico proposto, percebe-se que os graduandos dos três diferentes cursos, apesar de compreender a importância da integralidade do cuido, não acreditam em sua total aplicabilidade.

O modelo de assistência à saúde atual é voltado à um atendimento interdisciplinar, podendo apresentar variações, como pode ser verificado pelas percepções dos alunos que compuseram a amostra deste trabalho. Estas diversidades são naturais e esperadas, uma vez que a evolução científica como expressão máxima da racionalidade humana é processo progressivo de disciplinarização, pautado pelo conhecimento unificado e o saber especializado. Como consequência, a proposta interdisciplinar apresenta uma perspectiva de retrocesso (Hammes et al., 2020).

Este trabalho apresentou algumas limitações. As entrevistas foram realizadas em apenas uma clínica de ensino e apresentou um número reduzido de sujeitos pesquisados, podendo não refletir a realidade de demais processos de trabalho em equipe em locais como o selecionado, portanto, não sendo possível sua generalização. Contudo, os dados apresentados podem contribuir no incentivo a novas pesquisas sobre o cuidado interdisciplinar junto a referenciais teóricos consolidados na literatura científica, e, assim subsidiar maiores processos de reflexão para o próprio campo de estudo, sendo já um avanço positivo. Outro ponto a ser destacado, foi a escolha de uma teoria de enfermagem para fundamentar a prática baseada em evidências.

\section{Conclusão}

Este trabalho apresentou duas categorias temáticas: percepções sobre os processos de trabalho desenvolvidos dentro da clínica pelas equipes e percepção de um cuidado ideal. Os cursos envolvidos ainda não superaram o modelo tradicional constituído em núcleos, no qual a aprendizagem compartilhada ainda é segregada. A formação dos profissionais em sua prática, realizada de forma fragmentada e isolada, podem impedir o cuidado integral ao usuário, necessitando, portanto, de uma adequação ao perfil profissional da área da saúde em prol de um trabalho em equipe e na prática interprofissional.

Contudo, mesmo que ainda haja uma manutenção do modelo tradicional, os dados apresentados podem contribuir no incentivo a novas e constantes pesquisas sobre o cuidado interdisciplinar junto a referenciais teóricos consolidados na literatura científica, e, assim subsidiar mais processos de reflexão neste campo de estudo.

Estudos que auxiliem a composição de estratégias de identificação de modelos adequados à assistência em saúde são fundamentais, uma vez que auxiliam na condução e vivências realizadas por todos os envolvidos em clínicas de ensino e profissionais nela inseridos.

\section{Referências}

Agreli, H. L. F (2017). Prática interprofissional colaborativa e clima do trabalho em equipe na APS. 262 fl. Tese. (Doutorado em Fundamentos e Práticas em Gerenciamento em Enfermagem) - Escola de Enfermagem, Universidade de São Paulo, São Paulo.

Almeida, R. G. S., \& Silva, C. B. G. (2019). Interprofessional Education and the advances of Brazil. Rev Latino-Am Enfermagem. 27 : e3152.

Brasil. (2012). Conselho Nacional de Saúde. Resolução $N^{\circ} 466$, de 12 de dezembro de 2012: diretrizes e normas reguladoras de pesquisas envolvendo seres humanos. Brasília: Ministério da Saúde.

Costa, M. A. A. (2017). Potência da Educação Interprofissional para o desenvolvimento de competências colaborativas no trabalho em saúde. In: ToassI RFC. (Org.) Interprofissionalidade e formação na saúde: onde estamos? Ramona Fernanda Ceriotti Toassi (org). Porto Alegre: Rede UNIDA.

Ely, L. I., \& Toassi, R. F. C. (2017). Atividade de ensino integradora dos currículos na graduação em saúde: entre o multiprofissional, o interdisciplinar e o interprofissional. In: Toassi, R. F. C. (Org.) Interprofissionalidade e formação na saúde: onde estamos? Ramona Fernanda Ceriotti Toassi (org). Rede UNIDA.

Farias, D. N., Ribeiro, K. S. Q. S., Anjos, U. U., \& Brito, G. E. G. (2018). Interdisciplinaridade e interprofissionalidade na estratégia saúde da família. Trab educ saúde. 16(1):141-162.

Ferraro, J. L. S., Ferri, M. S., \& Simões, M. G. (2017). Como opera a transdisciplinaridade? Uma aproximação possível com o discurso disciplinar. In: Gallon, M. S.; Dopico, S. I. B.; Rocha Filho, J. B. (Org.) Transdisciplinaridade no ensino das ciências/ organizadores: Mônica da Silva Gallon, Sabrina Isis Brugnarotto Dopico, João Bernardes da Rocha Filho. EDUNISC. 
Research, Society and Development, v. 10, n. 4, e31210414206, 2021 (CC BY 4.0) | ISSN 2525-3409 | DOI: http://dx.doi.org/10.33448/rsd-v10i4.14206

Hammes, L. J., Karpinski, R., Rambo, R. A., Zitkoski, J. J., Hammes, I. L., \& Fetter, S. A. (2020). Interdisciplinarity as a challenge in teacher education. Braz J Dev. 6(10):76054-76065.

Japiassu, H. (1976). Interdisciplinaridade e patologia do saber. Imago.

Levine, M. E. (1996). The Conservation Principles: A Retrospective. Nursing Science Quarterly. 9(1):38-41.

Lewgoy, A., Kuplich, N., Machado, R., Faber, T., \& Fagundes, M. (2019). A perspectiva interprofissional na residência integrada multiprofissional em saúde hospitalar. Clinical \& Biomedical Research, 39 (1).

Minayo, M. C. S. (2014). O desafio do conhecimento: pesquisa qualitativa em saúde. (14a ed.), Hucitec/Abrasco.

Oliveira, M. R., Almeida, P., Moreira, T. M. M., Torres, R. A. M. (2019). Sistematização da assistência de enfermagem: percepção e conhecimento da enfermagem Brasileira. Rev Bras Enferm. 72(6):1547-1553.

Oliveira, E. S. F, Baixinho, C. L., \& Presado, M. H. C. V. (2019). Pesquisa qualitativa em saúde: uma abordagem reflexiva. Rev Bras Enferm. 72(4):830-831.

Peduzzi, M. (2016). O SUS é interprofissional. Interface comun. Saúde Educ. 20(56):199-201.

Peduzzi, M. (2017). Educação interprofissional para o desenvolvimento de competências colaborativas em saúde. In: Toassi, R. F. C. (Org.)

Interprofissionalidade e formação na saúde: onde estamos? Ramona Fernanda Ceriotti Toassi (org). Rede UNIDA.

Peduzzi, M., Agreli, H. L. F., Silva, J. A. M., \& Souza, H. S. (2020) Trabalho em equipe: uma revisita ao conceito e a seus desdobramentos no trabalho interprofissional. Trab educ saúde. 18(s1).

Salviano, M. E. M., Nascimento, P. D. F. S., Paula, M. A., Vieira, C. S., Frison, S. S., Maia, M. A., Souza, K. V. \& Borges, E. L. (2016). Epistemologia do cuidado de enfermagem: uma reflexão sobre suas bases. Rev Bras Enferm. 69(6):1240-1245.

Trentini, M., Paim, L., \& Silva, D. M. G. V. (2017). O método da pesquisa convergente assistencial e sua aplicação na prática de enfermagem. Texto contexto enferm.

Wilk, S., Hamiaz, M. K., Amyot, D., Michalowski, W., Kuziemsky, C., Catal, N., Rosu, D., Carrier, M., \& Giffen, R. (2020). An ontology-driven framework to support the dynamic formation of an interdisciplinary healthcare team. Int J Med Inform. 136:104075. 